This item was submitted to Loughborough's Research Repository by the author.

Items in Figshare are protected by copyright, with all rights reserved, unless otherwise indicated.

\title{
A New Interpretation of The Keller-Segel Model Based on Multiphase Modelling
}

PLEASE CITE THE PUBLISHED VERSION

LICENCE

CC BY-NC-ND 4.0

\section{REPOSITORY RECORD}

Byrne, Helen M., and Markus R. Owen. 2019. "A New Interpretation of the Keller-segel Model Based on Multiphase Modelling". figshare. https://hdl.handle.net/2134/195. 


\section{A New Interpretation of The Keller-Segel}

\section{Model Based on Multiphase Modelling}

Received: date / Revised version: date - C Springer-Verlag 2004

Abstract. In this paper an alternative derivation and interpretation are presented of the classical Keller-Segel model of cell migration due to random motion and chemotaxis. A multiphase modelling approach is used to describe how a population of cells moves through a fluid containing a diffusible chemical to which the cells are attracted. The cells and fluid are viewed as distinct components of a two-phase mixture. The principles of mass and momentum balances are applied to each phase, and appropriate constitutive laws imposed to close the resulting equations. A key assumption here is that the stress in the cell phase is influenced by the concentration of the diffusible chemical.

By restricting attention to one-dimensional cartesian geometry we show how the model reduces to a pair of nonlinear coupled partial differential equations for the cell density and the chemical concentration. These equations may be written in the form of the Patlak-Keller-Segel model, naturally including density-dependent

Helen M Byrne: Centre for Mathematical Medicine, School of Mathematical Sciences, University of Nottingham, Nottingham NG7 2RD, UK. e-mail: helen. byrne@nottingham.ac.uk

Markus R Owen: Department of Mathematical Sciences, Loughborough University, Loughborough, LE11 3TU, UK. e-mail: M.R.Owen@lboro.ac.uk.

Send offprint requests to: Helen M Byrne

Key words: Chemotaxis - Keller-Segel - Multiphase - Pattern formation 
nonlinearities in the cell motility coefficients. There is a direct relationship between the random motility and chemotaxis coefficients, both depending in an inter-related manner on the chemical concentration. We suggest that this may explain why many chemicals appear to stimulate both chemotactic and chemokinetic responses in cell populations.

After specialising our model to describe slime mold we then show how the functional form of the chemical potential that drives cell locomotion influences the ability of the system to generate spatial patterns. The paper concludes with a summary of the key results and a discussion of avenues for future research.

\section{Introduction}

The migratory behaviour of many cells is strongly influenced by the chemical composition of their microenvironment and this may, in turn, be regulated by the cells themselves. For example, during the aggregation phase of their life cycle, dictyostellium discoedeum secrete cAMP, a diffusible chemical to which the slime mold cells are attracted [17]. Equally, during angiogenesis in healing wounds and tumours, endothelial cells from the intact vasculature migrate specifically towards low oxygen regions, their speed and direction of motion being modulated by chemicals, including vascular endothelial growth factor, that are secreted by white blood cells such as macrophages that have already localised at the wound or tumour site $[7,19]$.

One of the most widely used experimental assays for testing the impact of specific chemicals on cell migration is the Boyden chamber assay $[8$, 11,25]. Here cells are placed on top of a porous filter within which a prescribed chemical distribution (usually either spatially uniform or varying 
monotonically with depth) has been established. By studying the impact that the concentration and spatial distribution of the chemical have on the speed with which the cells migrate through the filter it is possible to characterise chemosensitive cell movement. It is not surprising that a variety of mathematical approaches have been used to describe this phenomenon. For example, Stokes and Lauffenburger [58] used a stochastic model, Anderson and Chaplain [4] developed a hybrid probabilistic model to study endothelial cell migration during angiogenesis, and Dallon and Othmer [15] developed discrete cellular models to describe slime mold.

Probably the most widely used mathematical framework for studying directed cell motion is based on the Patlak-Keller-Segel model [32,33,47], a continuum model which, assuming one-dimensional Cartesian geometry, may be stated as follows:

$$
\begin{gathered}
\frac{\partial n}{\partial t}=\frac{\partial}{\partial x}\left(\mu(n, a) \frac{\partial n}{\partial x}\right)-\frac{\partial}{\partial x}\left(\chi(n, a) n \frac{\partial a}{\partial x}\right)+f(n, a), \\
\frac{\partial a}{\partial t}=D_{a} \frac{\partial^{2} a}{\partial x^{2}}+g(n, a) .
\end{gathered}
$$

In equations $(1,2), n(x, t)$ represents the density of the cell population, $a(x, t)$ the chemoattractant concentration, $f$ and $g$ represent their respective rates of production, and $D_{a}$ the chemical's assumed constant diffusion coefficient. Chemosensitive movement is categorised in this framework as undirected (random movement, often referred to as chemokinesis) and chemotactic (directed movement up or down a chemical gradient), with coefficients dependent on $n$ and $a$ given by the functions $\mu(n, a)$ and $\chi(n, a)$ respectively. 
While the Patlak-Keller-Segel (henceforth, Keller-Segel) model may be presented as a phenomenological model, it may also be derived by taking a continuum limit of a biased or reinforced random walk $[2,44,53]$. The first rigorous derivation from a microscopic interacting particle model was carried out by Stevens [57]. In addition, many variants of the Keller-Segel model have been suggested. For example, in [52] Sherratt focuses on receptorchemical interactions in eukaryotic cells in order to derive an extension to the Keller-Segel model for cell movement. This extended model includes an additional evolution equation for the density of bound receptors per unit volume, and can be validated readily against experimental data. In [28] Hillen and Painter modify the Keller-Segel model by incorporating a celldensity dependent chemotaxis coefficient that prevents "overcrowding", or blow-up. These authors have recently applied a reinforced random walk approach to derive such motility coefficients [46]. In a similar vein, Primicerio and Zaltzman $[49,50]$ consider a Keller-Segel system with a chemotaxis coefficient that switches from one to zero at a threshold, and show that this can prevent blow-up.

In this paper we show how a variant of the Keller-Segel model may be derived by using a multiphase modelling approach $[16,20]$ to describe the migration of a population of cells through a fluid that contains a chemical to which the cells are attracted. The cells and fluid constitute distinct components of a two-phase mixture and the governing equations are derived by applying the principles of mass and momentum balance to each phase and 
introducing appropriate constitutive laws to describe, for example, the way in which the diffusible chemical influences the stress in the cellular phase.

Multiphase models have wide-ranging applications and were originally used for industrial applications such as fluidised beds, the deformation of fibre-reinforced materials such as tyres, and composites manufacturing $[1$, $18,55,56]$. Most biomedical applications have focussed on modelling the response of bones and cartilage to loading $[5,30,37,41]$ and, to a lesser extent, soft tissues such as heart [54] and brain [43]. A key feature of such models is the assumption that growth may be neglected so that there is no mass exchange (e.g. due to chemical reaction for industrial applications, or cell proliferation and death for biomedical applications). Recently, several authors have developed two- and three-phase models of solid tumour growth in which this assumption is relaxed so that mass exchange may occur $[10,13,14,38,48]$. In this paper we adopt a similar approach but, as stated above, we focus on the impact on cell migration of a diffusible chemical (the chemoattractant) that is distributed within the fluid phase.

Other features of our model include the natural appearance of densitydependent nonlinearities in the random motility and chemotaxis coefficients. Further, there is a direct relationship between the chemotaxis and random motion coefficients and we suggest that this may explain why many chemicals appear to stimulate chemokinetic and chemotactic responses in cell populations. We remark that a similar connection was derived by Keller and Segel [33]. Furthermore, Othmer and Stevens [44] obtained related motility 
coefficients by treating cell locomotion as a random reinforced walk and taking a continuum limit of the resulting discrete equations. In consequence, their physical interpretation of the random motility and chemotaxis coefficients complements ours. Density dependent chemotaxis has also been investigated by Hillen and Painter [28], and in a recent paper density dependence for both random motion and chemotaxis have been derived from a reinforced random walk with volume-filling [46].

The remainder of the paper is organised in the following way. In section 2 we show how a multiphase approach may be used to derive a variant of the Keller-Segel model. The model is specialised in section 3 to describe spatiotemporal pattern formation in slime mold. Attention focusses on how these results are affected by the manner in which we model the drag coefficient between the cells and fluid and the influence that the diffusible chemical has on the stress in the cell phase. The paper concludes in section 4 with a summary and discussion of our main results.

\section{Model Formulation}

In this section we develop our mathematical model for the growth and movement of a colony of cells. The governing equations are constructed by applying mass and momentum balances to the two constituent phases, namely the cells and the water. We assume that a cell-derived chemical is distributed within the water phase and that it modulates cell motility. The approach we adopt is similar to that presented in $[10,13,14,40]$. However, by assuming that the stress in the cellular phase is influenced by the chemical 
concentration we are able to provide a new interpretation of chemicallyregulated cell migration.

\subsection{Model Derivation}

In this section we use the principles of mass and momentum balance to develop a two-phase model that describes the evolution of a population of cells, in the presence of a chemical that diffuses through the water phase. For simplicity we restrict attention to one-dimensional cartesian geometry, so that the dependent variables are functions of time $t>0$ and position $x \in(-L, L)$ where $L$ denotes the domain half-width.

We denote by $n(x, t)$ and $w(x, t)$ the volume fractions occupied by the cells and water respectively, and by $v_{n}(x, t), v_{w}(x, t)$ and $\sigma_{n}(x, t), \sigma_{w}(x, t)$ the corresponding velocities and stresses (in one-dimension, these are scalar quantities). We also introduce $p(x, t)$ to represent the pressure and $a(x, t)$ to denote the concentration of the cell-derived diffusible chemical. We note here that $a(x, t)$ is measured in moles per unit volume, and that the chemical concentration in the water phase is given by $a / w$.

2.1.1. Mass Balance Equations Applying mass balances to the cell and water phases respectively, and assuming that the net rates $S_{n}$ and $S_{w}$ at which the two phases are produced are functions of $n, w$ and $a$, we deduce that

$$
\begin{aligned}
\frac{\partial n}{\partial t}+\frac{\partial}{\partial x}\left(v_{n} n\right) & =S_{n}(n, w, a) \\
\frac{\partial w}{\partial t}+\frac{\partial}{\partial x}\left(v_{w} w\right) & =S_{w} \equiv-S_{n}
\end{aligned}
$$


In equations (3)-(4) we have assumed that there is no local source of material to the system: cells proliferate and grow by absorbing water while water is liberated when cells die. We note also that in equations (3)-(4) we have assumed that the densities of the water and cells are constant and equal to one, without loss of generality: in practice, since cells are predominantly composed of water, this should be a reasonable approximation.

Following $[10,13,14,61]$, we assume that the cells and water form an incompressible, continuous material, with no voids present, so that

$$
n+w=1
$$

We suppose that, once it is in the water phase, the chemical $a(x, t)$ can not cross the cells' membranes and, hence, that it moves by a combination of advection with the water velocity $v_{w}$ and diffusion. Accordingly we assume that the evolution of the diffusible chemical $a(x, t)$ is governed by the following reaction-diffusion-advection equation:

$$
\frac{\partial a}{\partial t}+\frac{\partial}{\partial x}\left(a v_{w}\right)=D_{a} \frac{\partial^{2} a}{\partial x^{2}}+S_{a}(a, n)
$$

In (6), $D_{a}$ represents the assumed constant diffusion coefficient of the chemical in water and $S_{a}(a, n)$ the net rate at which it is produced by the cells.

2.1.2. Momentum Balance Equations Neglecting inertial effects and assuming that there are no external body forces, the momentum equations 
for the cell and water phases read

$$
\begin{aligned}
& 0=\frac{\partial}{\partial x}\left(n \sigma_{n}\right)+F_{n w}+p \frac{\partial n}{\partial x}, \\
& 0=\frac{\partial}{\partial x}\left(w \sigma_{w}\right)-F_{n w}+p \frac{\partial w}{\partial x},
\end{aligned}
$$

where $F_{n w}$ denotes the force, or drag, that the water phase exerts on the cells (the cells exert an equal and opposite force, $-F_{n w}$, on the water phase). The terms $p \frac{\partial n}{\partial x}$ and $p \frac{\partial w}{\partial x}$ are interfacial forces that arise from the averaging process (for details, see [16]).

To close equations (3)-(8), it remains to specify the functions $S_{i}(i, n, m, a)$, the drag term $F_{n w}$, the stresses $\sigma_{i}(i=n, w)$ and appropriate boundary and initial conditions. A discussion of the boundary and initial conditions and the source terms is postponed to section 2.2 while constitutive laws for the drag terms and stresses are included below.

\subsubsection{Constitutive Laws We motivate our specification of $\sigma_{n}$ and $\sigma_{w}$ in (7)} and (8) as follows. We view the water as an isotropic fluid, with pressure $p$. While the cells are predominantly composed of water, their behaviour is more complex: they are assumed to alter their motility in response to the local concentration of the chemical $a(x, t)$, such sensitivity most likely being regulated by receptors on the cell membrane. In addition, even in the absence of the chemical $a$, the cells may explore their environment by extending and retracting pseudopodia, and alter their behaviour in an appropriate manner $[10,13]$. For example, if the cells are so sparsely populated that their pseudopodia do not meet the cells may be unable to detect each 
other and, hence, do not alter their behaviour. By contrast, if the cells are too densely populated they may repel each other. We introduce the potential function $\Lambda(n, a)$ to account for the way in which the cells' behaviour differs from that of the bulk water. Thus we write:

$$
\sigma_{n}=-p-\Lambda(n, a), \quad \sigma_{w}=-p .
$$

In section 4 we show how the choice of the potential function $\Lambda(n, a)$ affects the behaviour of the cells. We remark that more complex models could be used to describe the water and cell phases. For example, the extracellular fluid may behave as a viscous fluid and the cells as a visco-elastic material $[21,22,36,39]$. We restrict attention to highly idealised cases in this paper in order to focus on the effect that the chemical $a(x, t)$ has on cell migration.

Following $[10,13]$, we assume that the drag terms are proportional to the relative velocities of the two phases, with a constant of proportionality $\hat{k}=\hat{k}(n, w)$ that depends on the composition of the material, so that, using $(5)$,

$$
F_{n w}=\hat{k}(n, w)\left(v_{w}-v_{n}\right) \equiv k(n)\left(v_{w}-v_{n}\right) .
$$

Before outlining how our two phase model be reduced to a pair of coupled partial differential equations for $n$ and $a$, we first remark upon the way in which we have incorporated cell migration into our model. Obviously the mechanisms that regulate the movement of different types of cells and/or simple motile organisms are cell- (or organism-) specific. For ex- 
ample, leukocytes migrate towards a site of infection by crawling over the underlying tissue matrix $[9,11,25]$ whereas bacteria such as Escherichia coli swim by a combination of advection and rotation $[6,9]$ and other bacteria such as Euglena gracilis swim towards a light-source by beating their flagella in an appropriate manner $[26,27]$. When developing our model we have not considered the details of how the cells move: instead we have focussed on the role played by the diffusible chemical. In principle it would be possible to extend our model to account for details of cell migration. For example, we could model leukocyte migration through tissue by including a third phase to describe the extracellular matrix and additional constitutive laws to describe its mechanical properties, the rate at which it is produced and the interaction forces between the tissue matrix, the cells and the water. We postpone such extensions for future work.

\subsection{Model Simplification}

In this section we show how the two-phase model developed in section 2.1 can be reduced to a nonlinear parabolic equation for the cell phase $n(x, t)$ coupled to a reaction-diffusion-advection equation for the chemical $a(x, t)-$ this model is our modified version of the Keller-Segel model for cell migration. In order to perform this reduction, we must derive expressions relating the other dependent variables to $n(x, t)$ and $a(x, t)$.

Adding equations (7) and (8) we obtain the overall system force balance 


$$
0=\frac{\partial}{\partial x}\left(n \sigma_{n}+w \sigma_{w}\right)=-\frac{\partial}{\partial x}(p+n \Lambda) .
$$

Integrating this equation with respect to $x$ we deduce that the pressure $p$ satisfies

$$
p=-n \Lambda+P(t)
$$

where $P(t)$ is an arbitrary function of time, determined (in principle) by imposing the initial conditions (since, in practice, only pressure-gradients influence the system behaviour, we need not specify $P(t))$.

Adding equations (3) and (4), noting that $n+w=1$ (see equation (5)) and integrating with respect to $x$, we deduce further that the velocities $v_{n}$ and $v_{w}$ are related in the following way:

$$
n v_{n}+w v_{w}=0
$$

In equation (12) we have assumed that the system is symmetric about $x=0$ and hence that $v_{n}=v_{w}=0$ there. This would follow, for example, from imposing symmetry boundary conditions.

Substituting for $\sigma_{w}$ and $F_{n w}$ in the momentum balance for the water phase, equation (8), we deduce

$$
0=w \frac{\partial p}{\partial x}+k(n)\left(v_{w}-v_{n}\right)
$$

Substituting for $p$ and $v_{w}$ from (11) and (12), and using (5), we obtain the following expression for $v_{n}$ in terms of $n$ and $a$ : 


$$
v_{n}=-\frac{(1-n)^{2}}{k(n)} \frac{\partial}{\partial x}(n \Lambda)
$$

Finally substituting for $v_{n}$ in (3) we deduce that the cell phase $n(x, t)$ evolves in the following manner:

$$
\frac{\partial n}{\partial t}=\frac{\partial}{\partial x}\left\{\frac{n(1-n)^{2}}{k(n)} \frac{\partial}{\partial x}(n \Lambda(n, a))\right\}+S_{n}(n, 1-n, a) .
$$

Thus our reduced model comprises equations (14) and (6) which define the evolution of the dependent variables $n(x, t)$ and $a(x, t)$. We study (14) and (6) with no-flux boundary conditions at $x=0, L$ and initial distributions for $n$ and $a$ prescribed, so that:

$$
\begin{gathered}
\frac{n(1-n)^{2}}{k(n)} \frac{\partial(n \Lambda)}{\partial x}=\frac{\partial a}{\partial x}=0 \quad \text { at } x=0, L, \\
n(x, 0)=n_{0}(x), \quad a(x, 0)=a_{0}(x) .
\end{gathered}
$$

We remark that, since $v_{w}=0$ at $x=0, L$, the second of equations (15) guarantees no flux of $a$ at $x=0, L$.

Before analysing our reduced model, we pause to make several remarks. Firstly, while the reduced model is formulated in terms of $n$ and $a$, the other dependent variables $\left(p, w, v_{n}\right.$ and $\left.v_{w}\right)$ that were introduced to develop the model can be expressed in terms of $n$ and $a$. In particular, we have

$$
\begin{aligned}
p=-n \Lambda+P(t), & w=1-n, \\
v_{n}=-\frac{(1-n)^{2}}{k(n)} \frac{\partial}{\partial x}(n \Lambda), & v_{w}=\frac{n(1-n)}{k(n)} \frac{\partial}{\partial x}(n \Lambda) .
\end{aligned}
$$


We remark that the system $(6,14)$ can be derived by considering a three phase mixture of cells, water and chemical, where the chemical phase is assumed to have negligible mass. While more involved, this approach has the advantage that it uncovers the functional form of the chemical diffusion coefficient and, in particular, its dependence on the drag coefficient between the water and the chemical. In the interests of brevity, we omit the threephase derivation here. We note also that in our model derivation, the impact of osmosis on water transport has been neglected (details of how osmosis may be incorporated may be found in [37]).

\subsection{Comparison with Keller-Segel Model}

By using the chain rule and defining random motion and chemotaxic coefficients $\mu(n, a)$ and $\chi(n, a)$ appropriately, we can rewrite equation (14) in a form which more closely resembles the Keller-Segel model [33]:

$$
\frac{\partial n}{\partial t}=\frac{\partial}{\partial x}\left\{\mu(n, a) \frac{\partial n}{\partial x}-\chi(n, a) n \frac{\partial a}{\partial x}\right\}+S_{n}(n, 1-n, a)
$$

where

$$
\mu(n, a)=\frac{n(1-n)^{2}}{k(n)} \frac{\partial}{\partial n}(n \Lambda) \quad \text { and } \quad \chi(n, a)=-\frac{n(1-n)^{2}}{k(n)} \frac{\partial \Lambda}{\partial a} .
$$

Assuming $k(n)>0$, we deduce that for a well-posed problem, in which the chemical $a$ acts as a chemoattractant (rather than a chemo-repellent), the potential function $\Lambda(n, a)$ must satisfy

$$
\frac{\partial \Lambda}{\partial a}<0<\frac{\partial}{\partial n}(n \Lambda)
$$


Biologically, the first condition, $\frac{\partial \Lambda}{\partial a}<0$, means that the pressure in the cell phase is relieved by movement up a chemical gradient. This pressure reduction may be due to increased adhesion of amoeboid cells to a substrate, or to reductions in effective temperature via reduced tumbling of swimming cells [39]. The second condition, $\frac{\partial}{\partial n}(n \Lambda)>0$, reflects the need to avoid "backward diffusion", in the absence of regularising terms such as viscosity [13].

Referring to equation (18) we note that if $\frac{\partial \Lambda}{\partial a} \neq 0$ then, in contrast to the standard Keller-Segel model, the chemical automatically exerts a dual effect on the cells' migration: it not only stimulates directed motion up spatial gradients but also alters the random motility coefficient $\mu(n, a)$. This result may explain why many chemicals appear to exert chemokinetic and chemotactic effects on cell migration $[12,45,52]$.

Using the definitions of $\mu(n, a)$ and $\chi(n, a)$ stated above, we can predict conditions under which our model reduces to a commonly used form of the Keller-Segel model $(1,2)$, in which the motility parameters do not depend on cell density $[42,44,52]$. In particular, if $k(n)$ and $\Lambda(n, a)$ are defined in the following manner

$$
k(n)=n(1-n)^{2} \quad \text { and } \quad \Lambda(n, a)=\Lambda(a)
$$

then

$$
\mu(n, a)=\mu(a)=\Lambda(a) \quad \text { and } \quad \chi(n, a)=\chi(a)=-\frac{\partial \Lambda}{\partial a} .
$$


Further, if $\Lambda(a)$ depends weakly on $a$ while $\frac{\partial \Lambda}{\partial a}$ is large, then we may have $\mu(n, a) \sim \Lambda_{0}$, a constant. This will be the case if, for example, $\Lambda(a)=$ $\Lambda_{0}+\Lambda_{1} \exp (-\lambda a)$ where $\Lambda_{1}, \lambda^{-1} \ll 1$ and $\Lambda_{0}, \Lambda_{1} \lambda \sim O(1)$.

Given that the cells are likely to sense and respond to the number of moles of chemical per unit volume of water rather than the number of moles per unit volume, it is likely that the chemotactic component of the potential should be of the form $\Lambda=\Lambda(a /(1-n))$. In this case it is not possible to recover density independent motility parameters.

By treating cell locomotion as a random reinforced walk and taking the continuum limit, Othmer and Stevens [44] derived the following master equation to describe cell migration:

$$
\frac{\partial n}{\partial t}=\frac{\partial}{\partial x}\left\{n \frac{\partial}{\partial x}\left(\ln \left(\frac{n}{\Phi(a)}\right)\right)\right\}
$$

where the function $\Phi(a)$ models the rate at which cells move to adjacent sites. We remark that, if we fix $n \Lambda=\ln (n / \Phi(a))$ and assume $k(n)=(1-n)^{2}$ (or, equivalently, in terms of the original variables, $\hat{k}(n, w)=w^{2}$ ), equation (14) reduces to (21).

In general, both the random motion and chemotaxis coefficients will contain nonlinear factors proportional to $n$ and $(1-n)$, which ensure that there is no cellular movement when $n=0$ or $n=1$. While some authors have incorporated such factors into models of cell migration, until very recently this has been done on a rather ad hoc basis. For example, Hillen and Painter [28] developed a model of cell migration in which the chemotaxis coefficient contained a factor $(1-n)$, but the random motion coefficient did not. More 
recently, the same authors have used a reinforced random walk approach to derive equations for chemosensitive movement that include such factors in both random motility and chemotaxis coefficients [46].

Here, as a result of using a multiphase modelling framework to derive the governing equations, we are able to relate the random motility coefficient $\mu(n, a)$ and the chemotaxis coefficient $\chi(n, a)$ to fundamental aspects of the cells' behaviour.

There is a wide variety of techniques that could be used to study our reduced model. For example, we could investigate whether the system supports steady travelling waves by seeking solutions of the form $a(x, t)=A(z)$ and $n(x, t)=N(z)$ where $z=x-c t$ is the travelling wave coordinate and $c$ is the wavespeed [42]. In order to gain insight into the long-time behaviour of the system, we may also examine the existence, uniqueness and stability of steady solutions.

As a starting point for a more detailed model analysis, in the next section we investigate the ability of our reduced model, specialised to describe slime mold aggregation, to generate spatial patterns.

\section{Application to Spatial Pattern Formation in Slime Mold}

We now investigate the ability of our reduced model $(14,6)$ to generate spatial patterns, paying particular attention to how this is affected by the choices of the drag coefficient $k(n)$ and the potential function $\Lambda(n, a)$. In order to simplify the analysis and to facilitate comparison with existing models we first specialise our model to describe slime mold aggregation [42, 
51]. Accordingly we assume that the rates of cell proliferation and cell death are negligible, that each cell produces chemical at a constant rate $s_{0}$ and that the chemical decays with time constant $s_{1}^{-1}$. Under these assumptions equations (14) and (6) become

$$
\begin{gathered}
\frac{\partial n}{\partial t}=\frac{\partial}{\partial x}\left(\frac{n(1-n)^{2}}{k(n)} \frac{\partial}{\partial x}(n \Lambda)\right) \\
\frac{\partial a}{\partial t}+\frac{\partial}{\partial x}\left(\frac{a n(1-n)}{k(n)} \frac{\partial}{\partial x}(n \Lambda)\right)=D_{a} \frac{\partial^{2} a}{\partial x^{2}}+s_{0} n-s_{1} a .
\end{gathered}
$$

We solve equations (22) and (23) subject to (15)-(16). First, however, we recast the model in terms of dimensionless variables. Scaling distance with the domain length $L$ and time with the chemical diffusion timescale $L^{2} / D_{a}$ and using * to denote dimensionless variables, we introduce

$$
\begin{aligned}
x^{*} & =\frac{x}{L}, \quad t^{*}=\frac{D_{a} t}{L^{2}}, \quad n^{*}=n, \quad a^{*}=\frac{s_{0} L^{2} a}{D_{a}}, \\
k^{*}\left(n^{*}\right) & =k(n), \quad \Lambda^{*}\left(n^{*}, a^{*}\right)=\frac{\Lambda(n, a)}{D_{a}}, \quad s_{1}^{*}=\frac{s_{1} D_{a}}{L^{2}} .
\end{aligned}
$$

We remark that the cell volume fraction is a dimensionless quantity and hence we do not rescale $n$. The typical chemical concentration that is used to scale $a$ is the maximum chemical concentration that can be produced (by the cells) during the chosen timescale, the chemical diffusion timescale.

Rewriting equations $(22,23)$ in terms of dimensionless variables we obtain (omitting *s for clarity)

$$
\begin{aligned}
\frac{\partial n}{\partial t} & =\frac{\partial}{\partial x}\left(\frac{n(1-n)^{2}}{k(n)} \frac{\partial}{\partial x}(n \Lambda)\right) \\
\frac{\partial a}{\partial t}+\frac{\partial}{\partial x}\left(\frac{a n(1-n)}{k(n)} \frac{\partial}{\partial x}(n \Lambda)\right) & =\frac{\partial^{2} a}{\partial x^{2}}+n-s_{1} a
\end{aligned}
$$


The boundary and initial conditions are now given by

$$
\begin{gathered}
\frac{n(1-n)^{2}}{k(n)} \frac{\partial(n \Lambda)}{\partial x}=\frac{\partial a}{\partial x}=0 \quad \text { at } \quad x=0,1 \\
n(x, 0)=n_{i n}(x), \quad a(x, 0)=a_{i n}(x),
\end{gathered}
$$

where $n_{i n}(x)$ and $a_{i n}(x)$ are prescribed functions.

\subsection{Necessary Conditions for Pattern Formation}

In this section we investigate the stability of the spatially-uniform steady states of equations (24) and (25). By generalising Turing's criteria for diffusion driven instability in systems of coupled reaction diffusion equations [42, $60]$, we derive conditions under which the spatially-uniform steady states are stable to time-dependent perturbations and unstable to spatio-temporal perturbations.

Setting $\frac{\partial}{\partial t}=0=\frac{\partial}{\partial x}$ in equations (25) and (24) we deduce that at the spatially-uniform steady state $\left(n_{s}, a_{s}\right)$

$$
\left(n_{s}, a_{s}\right)=\left(n_{0}, n_{0} / s_{1}\right)=\left(n_{0}, a_{0}\right)
$$

where the parameter $n_{0}>0$ may vary.

We linearise about this steady state by introducing $0<\epsilon \ll 1$ and seeking solutions of the form

$$
n=n_{0}+\epsilon n_{1}(x, t)+O\left(\epsilon^{2}\right), \quad a=a_{0}+\epsilon a_{1}(x, t)+O\left(\epsilon^{2}\right),
$$

assuming that the initial conditions are compatible with such a power series expansion. 
Equating to zero coefficients of $O(\epsilon)$ we deduce that $n_{1}(x, t)$ and $a_{1}(x, t)$ satisfy

$$
\begin{aligned}
\frac{\partial n_{1}}{\partial t}= & \frac{n_{0}\left(1-n_{0}\right)^{2}}{k_{0}}\left\{\left(\Lambda_{s}+n_{0} \Lambda_{n}\right) \frac{\partial^{2} n_{1}}{\partial x^{2}}+n_{0} \Lambda_{a} \frac{\partial^{2} a_{1}}{\partial x^{2}}\right\} \\
\frac{\partial a_{1}}{\partial t}= & \frac{\partial^{2} a_{1}}{\partial x^{2}}-\frac{a_{0} n_{0}\left(1-n_{0}\right)}{k_{0}}\left(\left(\Lambda_{s}+n_{0} \Lambda_{n}\right) \frac{\partial^{2} n_{1}}{\partial x^{2}}+n_{0} \Lambda_{a} \frac{\partial^{2} a_{1}}{\partial x^{2}}\right) \\
& +n_{1}-s_{1} a_{1},
\end{aligned}
$$

where $k_{0}=k_{n w}\left(n=n_{0}\right), \Lambda_{s}=\Lambda\left(a_{s}, n_{s}\right), \Lambda_{n}=\frac{\partial \Lambda}{\partial n}\left(a_{s}, n_{s}\right)$ and $\Lambda_{a}=$ $\frac{\partial \Lambda}{\partial a}\left(a_{s}, n_{s}\right)$.

We seek solutions of (29)-(30) of the form

$$
\left(n_{1}, a_{1}\right)=e^{i \lambda x+\rho t}(\mathcal{N}, \mathcal{A})
$$

where $\lambda$ denotes the wave number of a particular spatial perturbation and $\rho=\rho(\lambda)$ represents its growth rate. The underlying steady state is said to be unstable to spatial perturbations if there exists $\lambda \neq 0$ such that $\Re(\rho(\lambda))>0$.

In order to satisfy the no-flux boundary conditions imposed at $x=0,1$ we require $\lambda=j \pi(j=0,1,2, \ldots)$. Substituting with (31) in (29) and (30) we deduce further that for nontrivial solutions (ie $\mathcal{N}, \mathcal{A} \neq 0$ ), $\rho(\lambda)$ satisfies the following dispersion relation

$$
0=\rho^{2}+B\left(\lambda^{2}\right) \rho+C\left(\lambda^{2}\right),
$$

where

$$
\begin{aligned}
& B\left(\lambda^{2}\right)=s_{1}+\left(1+\frac{n_{0}\left(1-n_{0}\right)^{2}}{k_{0}}\left(\Lambda_{s}+n_{0} \Lambda_{n}\right)-\frac{a_{0} n_{0}^{2}\left(1-n_{0}\right) \Lambda_{a}}{k_{0}}\right) \lambda^{2} \\
& C\left(\lambda^{2}\right)=\frac{n_{0}\left(1-n_{0}\right)^{2}}{k_{0}}\left(n_{0} \Lambda_{a}+s_{1}\left(\Lambda_{s}+n_{0} \Lambda_{n}\right)+\left(\Lambda_{s}+n_{0} \Lambda_{n}\right) \lambda^{2}\right) \lambda^{2} .
\end{aligned}
$$


Note that the steady state is neutrally stable to uniform perturbations $(\lambda=$

0 ), because of the factor $\lambda^{2}$ in $C\left(\lambda^{2}\right)$ - this arises because there are no source terms in (24).

We anticipate (see (20)) that $\Lambda_{s}+n_{0} \Lambda_{n}>0>\Lambda_{a}$, and $n_{0}\left(1-n_{0}\right)^{2} / k_{0}>$ 0 . Therefore, in equation (32), $B\left(\lambda^{2}\right)>0$ for all $\lambda \geq 0$ since $s_{1}>0$, by assumption. Consequently, a necessary condition for instability of the underlying steady state is that $C\left(\lambda^{2}\right)<0$ for some choice of $\lambda$. This will be the case if

$$
n_{0} \Lambda_{a}+s_{1}\left(\Lambda_{s}+n_{0} \Lambda_{n}\right)<0
$$

When (33) is satisfied, instability is predicted for the following range of $\lambda$ :

$$
0<\lambda^{2}=j^{2} \pi^{2}<-\left(s_{1}+\frac{n_{0} \Lambda_{a}}{\Lambda_{s}+n_{0} \Lambda_{n}}\right) .
$$

Note that the condition for a patterning instability is equivalent to that derived by Keller and Segel in their original paper on slime mold aggregation [32]. The equivalence is established by (19), which defines random motion and chemotaxis coefficients in terms of $\Lambda(n, a)$ and $k(n)$. Substituting the relevant terms into equation (4.15) of [32], the common factor $n_{0}\left(1-n_{0}\right)^{2} / k_{0}$ cancels throughout provided it is non-zero, to give (33). The analysis of Painter and Hillen is also equivalent in the case of zero cell kinetics and one chemical species (section 5.1, Case 1 in [46]). We remark that while the conditions for pattern formation (i.e. instability of the underlying steady state) are independent of the drag function $k(n)$ (see (33)), the growth rate of a given perturbation depends on the drag coefficient (see (32)). 


\subsection{Case Studies}

The above concepts are now illustrated by considering two choices for the potential function $\Lambda(n, a)$. In order to highlight the role played by the chemical $a$ in regulating cell migration, we consider only potential functions for which $\Lambda(n, a)=\Lambda(\hat{a})$ where $\hat{a}=a / w=a /(1-n)$ (i.e. the cells respond to the concentration of chemoattractant in water). Specifically, we consider:

$$
\Lambda(\hat{a})=\alpha_{1}+\beta_{1} e^{-\theta_{1} \hat{a}}
$$

and

$$
\Lambda(\hat{a})=\alpha_{2}+\frac{\beta_{2}}{1+\left(\theta_{2} \hat{a}\right)^{m}} .
$$

In $(35,36)$ the coefficients $\alpha_{i}, \beta_{i}$ and $\theta_{i}(i=1,2)$ and $m$ are assumed to be non-negative. In both cases the potential consists of a background (which stimulates a constant level of random movement) and a component which decreases as $\hat{a}=a /(1-n)$ increases.

To supplement our analysis, we constructed numerical simulations of $(24,25)$ using the NAG routine D03PCF. D03PCF uses a finite difference spatial discretisation and the method of lines, and integrates the resulting system of ODEs using a backward differentiation formula method. In all cases presented here we used a regular spatial grid with 1001 points, and a drag function $k(n)=n(1-n)$.

Exponential potential: $\Lambda(\hat{a})=\alpha_{1}+\beta_{1} e^{-\theta_{1} \hat{a}}$ 
In this case equation (33) is satisfied, and hence pattern formation may occur, if the model parameters satisfy the following inequality:

$$
\frac{\alpha_{1}}{\beta_{1}}<\left(\frac{\theta_{1} n_{0}}{s_{1}\left(1-n_{0}\right)^{2}}-1\right) e^{-\frac{\theta_{1} n_{0}}{s_{1}\left(1-n_{0}\right)}}
$$

By noting that the right-hand-side of (37) is bounded above by $e^{-2}(1+$ $\left.4 s_{1} / \theta_{1}\right)$ we deduce that no patterns will emerge if $\alpha_{1} / \beta_{1}>e^{-2}\left(1+4 s_{1} / \theta_{1}\right)$, regardless of the initial cell density $n_{0}$. By contrast, if $\alpha_{1} / \beta_{1}<e^{-2}(1+$ $\left.4 s_{1} / \theta_{1}\right)$ then spatial patterning is predicted for $n_{0} \in\left(n_{-}, n_{+}\right)$where $n_{ \pm}$ solve

$$
\frac{\alpha_{1}}{\beta_{1}}=\left(\frac{\theta_{1} n_{0}}{s_{1}\left(1-n_{0}\right)^{2}}-1\right) e^{-\frac{\theta_{1} n_{0}}{s_{1}\left(1-n_{0}\right)}}
$$

Thus, for this choice of $\Lambda(n, a)$ and viewing $n_{0}$ as a bifurcation parameter, there are threshold cell densities below and above which pattern formation is not predicted: if the cells are seeded too sparsely $\left(n_{0}<n_{-}\right)$or too densely $\left(n_{0}>n_{+}\right)$then the underlying spatially uniform steady state is linearly stable to periodic spatial perturbations. The range of cell densities for which spatial patterns are predicted widens as $\alpha_{1} / \beta_{1}$ decreases, and narrows as $s_{1} / \theta_{1}$ increases. We note that such lower and upper bounds on $n_{0}$ for patterning have been observed in experiments in which endothelial cells are plated at different densities on two-dimensional monolayers in vitro [3, 24]. When plated at low densities the cells are too sparsely populated to form a connected vascular network. Equally when they are plated at high densities there is insufficient free space available and the cells form dense patches, separated by holes. For intermediate plating densities a connected network 
of elongated endothelial cells forms. Ambrosi and coworkers [3,24] have developed an alternative, single phase model, based on Burger's equation, which successfully reproduces these phenomena. In contrast, the standard Keller-Segel model (1)-(2) with constant motility parameters gives only a lower bound on $n_{0}$ for patterns, although more complex functional forms for $\mu(a)$ and $\chi(a)$ such as those we use can give upper and lower bounds for patterning.

If there is no background potential $\left(\alpha_{1}=0\right)$, then spatial patterns may occur if $Q\left(n_{0}\right)=n_{0}^{2}-\left(2+\theta_{1} / s_{1}\right) n_{0}+1<0$. Since $Q(0)>0>Q(1)$ we deduce that $Q\left(n_{0}\right)$ has a unique root $n_{-} \in(0,1)$, and patterns may occur if $n_{0}>n_{-}$. In particular, there is a minimum cell density below which spatial patterns are not predicted but there is no upper-bound, beyond the physical constraint that $n_{0}<1$ (see equation (5)).

As patterns develop from perturbations to the homogeneous steady state, the problem becomes ill-posed if the random motility coefficient in equation (24) becomes negative, i.e. if $\Lambda+n \Lambda_{n}<0$ then we have backward diffusion. Intuitively, as the cells aggregate they reduce the water volume fraction, increasing the chemical concentration in the water, and hence relieving the cells' stress (this is the $n \Lambda_{n}$ contribution). If this effect is sufficient to counteract the positive term $\Lambda$, then it becomes favourable for cells to aggregate without any explicit chemotactic effect. This aggregation has no lower bound on wavelengths $\lambda$, and consequently unbounded growth rates [13]. For the problem to remain well-posed we require $\Lambda+n \Lambda_{n}>0$ at 
all solution points $(n(x, t), a(x, t))$, or equivalently

$$
\frac{\alpha_{1}}{\beta_{1}}>\left(\frac{\theta_{1} a}{(1-n)^{2}}-1\right) e^{-\frac{\theta_{1} a}{1-n}} .
$$

When $\alpha_{1}=0$, this simplifies to give $R(n, a)=n^{2}-\left(2+\theta_{1} a\right) n+1>0$. Since $R(0, a)>0>R(1, a)$ we deduce that, for a fixed value of $a, R(n, a)=0$ has a single root $n_{R}(a) \in[0,1]$ and the problem becomes ill-posed for $n>n_{R}(a)$. If, in addition, $a<1 / s_{1}$ then $R(n, a)>Q(n)$ and $n_{-}<n_{R}(a)$, so that a patterning bifurcation occurs for a smaller population density than that at which the system becomes ill-posed.

We summarise these results in Figure 1(a) where the patterning and ill-posed regions for a parameter set with $\alpha_{1}=0$ are illustrated. We note that the full range of $a_{0}$ for spatial patterning can be explored by varying $s_{1}$, since $a_{0}=n_{0} / s_{1}$ at the spatially-uniform steady state about which we linearise. A particular value of $s_{1}$ is identified with a particular line $a=n / s_{1}$, along which bifurcations to spatial patterns may occur. Clearly, as solutions evolve they may leave this line and/or leave the well-posed region. Indeed, simulations (not shown) with parameters corresponding to the asterisk in Figure 1(a) eventually evolve so that the cell population forms a sharp peak, and $(n, a)$ enters the ill-posed region for some point $(x, t) \in[0,1] \times(0, \infty)$. Thereafter the numerical simulations cease to be physically relevant.

In contrast, when a background potential is included $\left(\alpha_{1}>0\right)$, it is possible for solutions to remain well-posed for all time. Figure 1(b) illustrates how the patterning and ill-posed regions depicted in Figure 1(a) change when $\alpha_{1}>0$, and all other parameters are held fixed. For certain choices of 
$s_{1}$ the line $a=n / s_{1}$ lies outside the ill-posed region, and patterned solutions that lie sufficiently close to this line may remain well-posed for all time. This is confirmed by the simulations presented in Figure 2 which show how different initial conditions give rise to spatial patterns with different numbers of peaks. The point $\left(n^{*}(t), a^{*}(t)\right)$ at which a solution $(n(x, t), a(x, t))$ is closest to the ill-posed region may be defined by

$$
\left.\left\{\Lambda+n \Lambda_{n}\right\}\right|_{\left(n^{*}, a^{*}\right)}=\min _{x \in[0,1]}\left\{\Lambda+n \Lambda_{n}\right\} .
$$

Included on Figure 1(b) is the trajectory of this point from the simulation of Figure 2(a), illustrating that the solution does indeed remain within the well-posed region.

Comparing Figures 1(a) and (b) we can see that as $\alpha_{1}$ increases (and the strength of the constant background potential becomes more important) the size of the ill-posed region reduces and it is more likely that emerging spatial patterns will remain well-defined for all time. Equally, if $\alpha_{1}$ becomes too large then pattern formation is not possible because the chemotactic potential is dominated by the strong background signal.

Rational potential: $\boldsymbol{\Lambda}(\hat{\boldsymbol{a}})=\boldsymbol{\alpha}_{2}+\boldsymbol{\beta}_{2} /\left(\mathbf{1}+\left(\boldsymbol{\theta}_{2} \hat{\boldsymbol{a}}\right)^{m}\right)$

Here spatial patterning is predicted for

$$
\frac{\alpha_{2}}{\beta_{2}}<\left(\frac{m \theta_{2}^{m} n_{0}^{m}}{s_{1}^{m}\left(1-n_{0}\right)^{m}+\theta_{2}^{m} n_{0}^{m}} \frac{1}{1-n_{0}}-1\right) \frac{s_{1}^{m}\left(1-n_{0}\right)^{m}}{s_{1}^{m}\left(1-n_{0}\right)^{m}+\theta_{2}^{m} n_{0}^{m}}
$$

and the problem is well-posed for given solution pairs $(n, a)$ if

$$
\frac{\alpha_{2}}{\beta_{2}}>\left(\frac{m \theta_{2}^{m} a^{m}}{(1-n)^{m}+\theta_{2}^{m} a^{m}} \frac{n}{1-n}-1\right) \frac{(1-n)^{m}}{(1-n)^{m}+\theta_{2}^{m} a^{m}} .
$$


We first consider the case when $m=1$, and show why we expect patterning instability to lead to an ill-posed problem. Figure 3(a) shows an example of the patterning and ill-posed regions for $m=1$. It is clear that in the patterning regime, chemotactic aggregation can cause $n$ to become sufficiently large for the solution to enter the ill-posed region. Figure 3(b) shows a numerical simulation of just such aggregation - at time $t \approx 25$ the solution enters the ill-posed region.

With $m=1$ the condition for pattern formation reduces to

$P\left(n_{0}\right)=\left(1-\frac{\theta_{2}}{s_{1}}\right)\left(1+\frac{\alpha_{2}}{\beta_{2}}-\frac{\alpha_{2} \theta_{2}}{\beta_{2} s_{1}}\right) n_{0}^{2}-2\left(1+\frac{\alpha_{2}}{\beta_{2}}-\frac{\alpha_{2} \theta_{2}}{\beta_{2} s_{1}}\right) n_{0}+1+\frac{\alpha_{2}}{\beta_{2}}<0$.

It is straightforward to show that $P(0)>0, P(1)=\left(\alpha_{2} \theta_{2} / \beta_{2} s_{1}-1\right) \theta_{2} / s_{1}$, and that $P\left(n_{0}\right)$ has no turning points in the unit interval. If $\alpha_{2} \theta_{2} / \beta_{2} s_{1}>1$ then $P(1)>0$, and hence $P\left(n_{0}\right)>0$ for all $n_{0} \in[0,1]$ and no spatial patterns will occur. By contrast, if $\alpha_{2} \theta_{2} / \beta_{2} s_{1}<1$ then $P\left(n_{0}\right)$ has a unique root $n_{-} \in(0,1)$, such that $P\left(n_{0}\right)<0$ (and patterns are predicted) for $n_{0} \in\left(n_{-}, 1\right]$. In particular, with no background feedback $\left(\alpha_{2}=0\right)$ there is always a patterning bifurcation.

The well-posed condition for $m=1$ becomes

$T(n, a)=\left(1+\frac{\alpha_{2}}{\beta_{2}}\right) n^{2}-2\left(1+\frac{\alpha_{2}}{\beta_{2}}\right)\left(1+\theta_{2} a\right) n+\left(1+\theta_{2} a\right)\left(1+\frac{\alpha_{2}}{\beta_{2}}+\alpha_{2} \theta_{2} a\right)>0$

For fixed $a, T(0, a)>0, T(1, a)=\theta_{2} a\left(\alpha_{2} \theta_{2} a-\beta_{2}\right)$, and $T(n, a)$ has a critical point at $n>1$. Thus either $T(1, a)<0$ and there is exactly one 
root $n_{R}(a) \in[0,1]$, or $T(1, a)>0$ and there are no roots - notice that the former case always holds with no background feedback $\left(\alpha_{2}=0\right)$.

Fixing $s_{1}=n / a$, or equivalently $a=n / s_{1}$, it is clear that $T\left(n, n / s_{1}\right)>$ $P(n)$ so that $n_{-}<n_{R}(a)$. Furthermore, if $T\left(1,1 / s_{1}\right)=0$ then $P(1)=0$, which means that the patterning and well-posed boundaries always converge at $\left(n=1, a=1 / s_{1}=\beta_{2} /\left(\alpha_{2} \theta_{2}\right)\right)$ — incidentally they also converge at $(n=1, a=0)$. Consequently, for all values of $a$ the boundary of the ill-posed region lies between that for patterning and $n=1$, so that as cells aggregate and $n$ increases, we expect to cross that boundary $n_{R}(a)$. An example is illustrated in Figure 3 - this should be contrasted with Figures 1(b) and $4(\mathrm{~b})$.

These features with $m=1$ are very similar to the exponential case with no background (see Figure 1(a)), and mean that patterning occurs for $n_{0}>n_{-}$, but as solutions evolve they can cross the well-posed boundary $n_{R}(a)$

When $m \geq 2$ there are typically zero or two roots for the patterning bifurcation and the well- to ill-posed transition. Figure 4(a) shows an example of the patterning and well-posed regions for $m=2$. There are two threshold cell densities for patterning, $n_{ \pm}$, with patterning predicted for $n_{0} \in\left(n_{-}, n_{+}\right)$, and the ill-posed region sits inside this patterning domain. In the same way as for the exponential potential, the line $a=n / s_{1}$ can lie outside the ill-posed region, and patterns may develop and remain wellposed. Figure 4(b) shows a numerical simulation for this case, and part 
(a) includes the trajectory of the point in space closest to the ill-posed region, illustrating that the solution does indeed remain within the well-posed region.

\section{Conclusions}

In this paper we have shown how a variant of the Keller-Segel model of cell migration may be derived using a multiphase modelling framework. Features of our model include the appearance of cell-density dependent nonlinearities in the cell motility coefficients and a new explicit relationship between the random motility and chemotaxis coefficients, both depending in an inter-related manner on the concentration of the diffusible chemical. We suggest that this relationship may explain why many chemicals seem to elicit chemotactic and chemokinetic responses in cell populations. It would be relatively straightforward to test this model prediction. For example, we could simply fit our model, with a specific functional form of the chemical potential $\Lambda(n, a)$, to cell migration data obtained from Boyden chamber assays. By comparing the goodness of fit so obtained with that for a model in which the random motility and chemotaxis coefficients are independent functions we would then be able to establish the validity of our model.

We also observe that in general our approach leads to both upper and lower bounds for pattern formation. Similar upper and lower bounds have been observed experimentally, and found in a single phase model based on Burger's equation [3,24]. In contrast, the commonly studied Keller-Segel model $(1,2)$ with linear chemical kinetics and constant motility parameters 
gives only a lower bound on $n_{0}$ for patterns. It is the additional complexity given by non-constant functional forms for $\mu(n, a)$ and $\chi(n, a)$ that leads to the additional upper bound for patterning even with linear chemical source terms. These features are implicit in Keller and Segel's original work [32], and are also found in more recent papers [46]. Other results for chemotaxis systems with density dependent motility parameters include investigations of Lyapunov functions [29], and the prevention of blow-up $[28,49,50]$.

Obviously there are many ways in which the work in this paper could be extended. Systems with bounded chemotaxis have been shown to exhibit a coarsening effect similar to that seen in Cahn-Hilliard models $[28,46]$, and it is possible that such features may emerge from our model. Our simulations show some evidence of coarsening, but not necessarily to the extent that a single aggregation is seen (see Figure 2). It is possible that this may simply be because we have not continued our simulations for long enough. Further studies of the patterning process could include domain growth, and cell proliferation, which Painter and Hillen have shown can lead to the repeated insertion of new peaks [46].

We note that derivations based upon reinforced random walks generalise to higher dimensions. With a multiphase approach, the mass balance, momentum balance, and constitutive laws can be extended to higher dimensions, together with boundary conditions that specify zero normal velocity and zero normal stress. However, in general this will not allow for the re- 
duction to the Keller-Segel form, and the analysis and simulation of the full system will be an important challenge.

Apart from considering more realistic spatial geometries, there are several modelling assumptions that could be relaxed. For example, we could distinguish between cells that move by crawling over a substrate $[8,9]$ and organisms that swim by beating their flagella $[9,26,27]$. In order to describe cell crawling it would be necessary to introduce a third phase for the extracellular matrix (ECM) and to include additional constitutive laws to describe its mechanical properties and its interactions with the cells and water. In [31] a three-phase model involving cells, ECM and water is developed to show that ECM compression by an expanding tumour mass is more likely to explain the formation of collagenous capsules surrounding benign tumours than an immune response in which the host responds to the foreign body, or cancer, by expressing more ECM. The details of chemokine signalling could be included by considering ligand-receptor binding, and it would be interesting to try to relate such binding to cell adhesion and hence movement.

Other model modifications for which there is strong experimental evidence include assuming that the cell proliferation and death terms depend on the stress that the cells experience [14]. For example, osteoblasts within cartilage respond to moderate, periodic loading by producing more collagen whereas under excessive loading the mechanical integrity of the cartilage becomes compromised. 
We have seen in numerical simulations that $(24,25)$ can become ill-posed as $n$ increases due to the cellular aggregation induced by chemotaxis. We have considered $\Lambda(a, n)=\Lambda\left(\frac{a}{1-n}\right)$, for which the problem is well-posed if

$$
\frac{\partial}{\partial n}(n \Lambda)=\Lambda\left(\frac{a}{1-n}\right)+\frac{a n}{(1-n)^{2}} \Lambda^{\prime}\left(\frac{a}{1-n}\right)>0 \text {. }
$$

For $\Lambda^{\prime}<0$, as $n \rightarrow 1$ the system can become ill-posed, unless $\Lambda^{\prime} \rightarrow 0$ sufficiently fast. Intuitively this is because as cells aggregate they reduce the water volume fraction and hence effectively increase the chemical concentration. Solutions for some forms of $\Lambda$ do remain well-posed for certain initial conditions, but this seems to require certain properties — for example, a background potential may distinguish the cells from the water phase, introducing a non-zero diffusive component in the absence of any chemical stimulation. In practice we anticipate that cells cannot squeeze out all of the water, and that short range repulsive forces will come into play before then. Thus, one way to regularise the problem is to introduce an additional cellular potential dependent only on the cell density, reflecting the repulsion that cells have for each other when that density is too great:

$$
\Lambda(a, n)=\Lambda^{\text {chemo }}\left(\frac{a}{1-n}\right)+\Lambda^{\text {cells }}(n)
$$

For example, following [10] we may take

$$
\Lambda^{\text {cells }}= \begin{cases}\lambda \frac{n-n^{*}}{(1-n)^{2}} & \text { if } n \geq n^{*}, \\ 0 & \text { otherwise. }\end{cases}
$$

This gives an additional positive contribution to $\frac{\partial}{\partial n}(n \Lambda)$, which can reduce or completely eliminate the ill-posed region of the $(n, a)$ phase space. An 
example in which this cellular potential is included to maintain a well-posed problem is shown in Figure 5. Figure 5(a) shows the evolution of stable patterns, and part (b) shows the regions in phase space and the trajectory of the space point closest to the ill-posed region, defined by (39). Clearly the simulated solution no longer enters that region, and remains well-posed. Increasing the strength of the cellular potential completely eliminates the illposed region (not shown). An alternative approach, as demonstrated in [13], is to regularise the problem by including viscous effects in the constitutive laws for the cell stress.

In conclusion, in this paper we have shown how a multiphase modelling framework may be adapted to describe directed cell motion. The resulting models may be viewed as versions of the Keller-Segel model which has been widely used to study cell movement due to random motion and chemotaxis. In order to clarify the modelling process and, in particular, the manner in which the chemoattractant influences the stress experienced by the cells we considered simple cases in which the precise details of how the cells migrate (e.g. crawling or swimming) were neglected. In spite of its simplicity, our model yields a number of predictions that either agree with experimental results or could be tested against existing experimental data. For example, for certain choices of the chemical potential, we showed that spatial patterning could only occur if the initial cell density lay within a well-defined range. These results are consistent with experiments which show that endothelial cells on a two-dimensional monolayer will only form a connected network 
when the initial plating density lies within upper and lower limits $[3,24]$. In addition, our model formulation leads naturally to an explict relationship between the random motility and chemotaxis coefficients which could be tested against cell migration data from Boyden chamber assays $[8,11,25]$.

\section{References}

1. S.G. Advani (1994). Flow and rheology in polymeric composites manufacturing. Elsevier.

2. W. Alt (1980). Biased random walk models for chemotaxis and related diffusion approximations. J. Math. Biol 9: 147-177.

3. G. Serini, D. Ambrosi, E. Giraudo, A. Gamba, L. Preziosi and F. Bussolini (2003). Modeling the early stages of vascular network assembly. EMBO J. 22: 1771-1779.

4. A.R.A. Anderson and M.A.J. Chaplain (1998). Continuous and discrete mathematical models of tumour-induced angiogenesis. Bull. Math. Biol. 60: 857899.

5. M.K. Barker and B.B. Seedhom (1997). Articular cartilage deformation under physiological cycling loading, J. Biomech. 30: 377-381.

6. R.N. Bearon and T.J. Pedley (2000). Modelling run-and-tumble chemotaxis in a shear flow. Bull. Math. Biol. 62: 775-791.

7. N.T. Bennet and G.S. Schultz (1993). Growth factors and wound healing: Part II role in normal and chronic wound healing. The American Journal of Surgery 166: 74-81.

8. S.V. Boyden (1962). The chemotactic effect of mixtures of antibody and antigen on polymorphonuclear leukocytes. J. Exp. Med. 115: 453-466.

9. D. Bray (2001). Cell movements: from molecules to motility. Garland Publishing. 
10. C.J.W. Breward, H.M. Byrne and C.E. Lewis (2002). The role of cell-cell interactions in a two-phase of solid tumor growth, J. Math. Biol. 45: 125-152.

11. H.M. Buettner, D.A. Lauffenburger and S.H. Zigmond (1989). Measurement of leukocyte motility and chemotaxis parameters with the Millipore filter assay. J. Immunol. Meth. 123: 25-37.

12. H.M. Byrne, G. Cave and D.L.S. McElwain (1998). The effects of chemokinesis on leukocyte locomotion: a new interpretation of experimental results. IMA J. Math. Appl. Med. Biol. 15: 235-256.

13. H.M. Byrne, J.R. King, D.L.S. McElwain and L. Preziosi (2003). A two-phase model of solid tumor growth, Appl. Math. Lett.. 16: 567-573.

14. H.M. Byrne and L. Preziosi (2003). Modelling Solid Tumor Growth Using The Theory of Mixtures, IMA J. Math. Appl. Med. Biol.. 20: 341-366.

15. J.C. Dallon and H.G. Othmer (1997). A discrete cell model with adaptive signalling for aggregation of Dictyostelium discoideum. Phil. Trans Roy. Soc. B 352: 391-417.

16. D.A. Drew and L.A. Segel (1971). Averaged equations for two-phase flows. Stud. Appl. Math. 50: 205-231.

17. A. Early, T. Abe and J. William (1995). Evidence for positional differentiation of prestalk cells and for a morphogenetic gradient in dictyostelium. Cell 83: 91-99.

18. A. Farina and L. Preziosi (2000). Deformable porous media and composites manufacturing. In: Heterogeneous Media: Micromechanics, Modelling, Methods and Simulations, K. Markov and L. Preziosi, Eds., Birkhäuser.

19. J. Folkman and H. Brem (1992). Angiogenesis and inflammation. In: Inflammation: Basic Principles and Clinical Correlates, Second Edition. (eds. J.I. Gallin, I.M. Goldstein and R. Dnyderman), New York, Raven Press.

20. A.C. Fowler (1997). Mathematical models in the applied sciences. Cambridge University Press, Cambridge. 
21. S.J. Franks (2001). Mathematical modelling of tumour growth and stability. $\mathrm{PhD}$ thesis, University of Nottingham, England.

22. S.J. Franks and J.R. King (2003). Interactions between a uniformly proliferating tumour and its surroundings: uniform material properties. IMA J. Math. Med. Biol. (submitted).

23. Y.C. Fung (1990). Biomechanics: motion, flow, stress and growth. SpingerVerlag, New York.

24. A. Gamba, D. Ambrosi, A. Coniglio, A. de Candia, S. di Talia, E. Giraudo, G. Serini, L. Preziosi, and F. Bussolini (2003). Percolation, morphogenesis, and Burgers dynamics in blood vessel formation. Phys. Rev. Lett. 90 (11): 118101.

25. J.L. Haddox, R.R. Pfister and C.I. Sommers (1991). A visual assay for quantitating neutrophil chemotaxis in a collagen gel matrix. J. Immunol. Meth. 141: $41-52$.

26. D.P. Hader (1987). Polarotaxis, gravitaxis and vertical phototaxis in the green flagellate, Euglena-gracilis. Arch. Microbiol. 147: 179-183.

27. N.A. Hill and L.A. Plumpton (2000). Control strategies for the polarotactic orientation of the microorganism Euglena gracilis. J. theor. Biol. 203: 357365.

28. T. Hillen and K. Painter (2001). Global existence for a parabolic chemotaxis model with prevention of overcrowding. Adv. Appl. Math. 26: 280-301.

29. D. Horstmann (2001). Lyapunov functions and $L^{p}$-estimates for a class of reaction-diffusion systems. Coll. Math. 87: 113-127.

30. J.S. Hou, M.H. Holmes, W.M. Lai, and V.C. Mow (1989). Boundary conditions at the cartilage-synovial fluid interface for joint lubrication and theoretical verifications, J. Biomech. Eng. 111: 78-87.

31. T.J. Jackson and H.M. Byrne (2002). A mathematical model of tumour encapsulation, Math. Biosci. 180: 307-328. 
32. E.F. Keller and L.A. Segel (1970). Initiation of slime mold aggregation viewed as an instability. J. theor. Biol. 26: 399-415.

33. E.F. Keller and L.A. Segel (1971a). Model for chemotaxis. J. theor. Biol. 30: $225-234$.

34. E.F. Keller and L.A. Segel (1971b). Travelling bands of chemotactic bacteria: a theoretical analysis J. theor. Biol. 30: 235-248.

35. E.F. Keller (1980). Assessing the Keller-Segel model: how has it fared? In: Biological growth and spread, W. Jager, H. Rost and P. Tautu (Eds.), Lecture Notes in Biomathematics, 38: 379-387. Berlin, Springer-Verlag.

36. J.R. King and S.J. Franks (2003). Mathematical analysis of some multidimensional tissue growth models. Eur. J. Appl. Maths. (in press).

37. W.M. Lai, J.S. Hou and V.C. Mow (1991). A triphasic theory for the swelling and deformation behaviours of articular cartilage. J. Biomech. Eng. 113: 245258.

38. K. Landman and C.P. Please (2001). Tumor dynamics and necrosis: Surface tension and stability. IMA J. Maths. Appl. Med. Biol. 18: 131-158.

39. J. Lega and T. Passot (2003). Hydrodynamics of bacterial colonies: a model. Phys. Rev. E 67 (3): 31906.

40. S.R. Lubkin and T. Jackson (2002). Multiphase mechanics of capsule formation in tumours, J. Biomech. Eng. 124: 237-243.

41. V.C. Mow and W.M. Lai (1979). Mechanics of animal joints, Annu. Rev. Fluid Mech. 11: 247-288.

42. J.D. Murray (1989). Mathematical Biology. Springer-Verlag, New York.

43. C. Nicholson (1985). Diffusion from an injected volume of a substance in brain tissues with arbitrary volume fraction and tortuosity. Brain Res. 333: $325-329$.

44. H.G. Othmer and A. Stevens (1997). Aggregation, blowup and collapse: the ABCs of taxis in reinforced random walks. SIAM J. Appl. Math. 57: 1041- 
1081.

45. M.R. Owen and J.A. Sherratt (1997). Pattern formation and spatiotemporal irregularity in a model for macrophage-tumour interactions. J. theor. Biol. 189: $63-80$.

46. K.J. Painter and T. Hillen (2003). Volume-filling and quorum-sensing in models for chemosensitive movement, Can. App. Math. Quart. to appear.

47. C. Patlak (1953). Random walk with persistence and external bias. Bull. Math. Biophys. 15: 311-338.

48. C.P. Please, G. Pettet and D.L.S. McElwain (1998). A new approach to modelling the formation of necrotic regions in tumors, Appl. Math. Lett., 11: 89-94.

49. M. Primicerio and B. Zaltzman (2002). A free boundary problem arising in chemotaxis. Adv. Math. Sci. Appl. 12: 685-708.

50. M. Primicerio and B. Zaltzman (2002). Free boundary in radial-symmetric chemotaxis Proceedings WASCOM 2001, World Scientific.

51. L.A. Segel (1977). A theoretical study of receptor mechanisms in bacterial chemotaxis. SIAM J. Appl. Math. 32: 653-665.

52. J.A. Sherratt, E.H. Sage and J.D. Murray (1993). Chemical Control of Eukaryotic Cell Movement: A New Model. J. Theor. Biol. 162: 23-40.

53. B.D. Sleeman and H.A. Levine (1997). A system of reaction diffusion equations arising in the theory of reinforced random walks. SIAM J. Appl. Math. 57: $683-730$

54. S. Sorek and S. Sideman (1986). A porous medium approach for modelling heart mechanics: Part 1 theory, Math. Biosci. 81: 1-14.

55. A.J.M. Spencer (1972). Deformations of fibre-reinforced materials. Clarendon Press.

56. A.J.M. Spencer (1984). Continuum theory of the mechanics of fibre-reinforced composites. Springer Verlag. 
57. A. Stevens (2000). The derivation of chemotaxis equations as limit dynamics of moderately interacting stochastic many-particle systems. SIAM J. Appl. Math. 61: 183212.

58. C.I. Stokes and D.A. Lauffenburger (1991). Analysis of the roles of microvessel endothelial cell random motility and chemotaxis in angiogenesis. J. theor. Biol. 152: 377-403.

59. R. Tyson, S.R. Lubkin and J.D. Murray (1999). Model and analysis of chemotactic bacterial patterns in a liquid medium, J. Math. Biol. 38, 359-375.

60. A.M. Turing (1952). The chemical basis of morphogenesis. Phil. Trans. Roy. Soc. Lond. B237: 37-72.

61. J.P. Ward and J.R. King (1997). Mathematical modelling of avascular-tumour growth. IMA. J. Math. Appl. Med., 14: 39-69. 


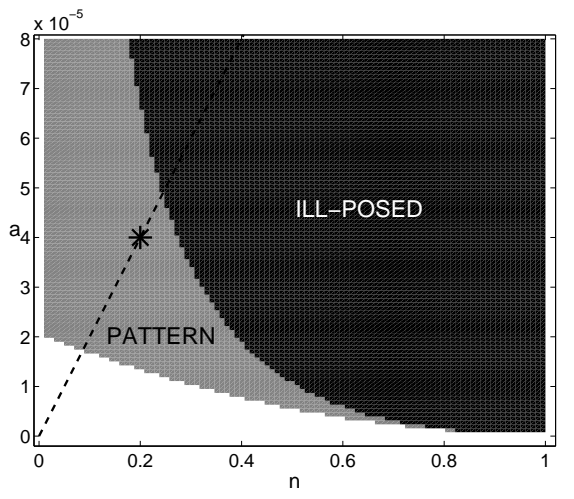

(a)

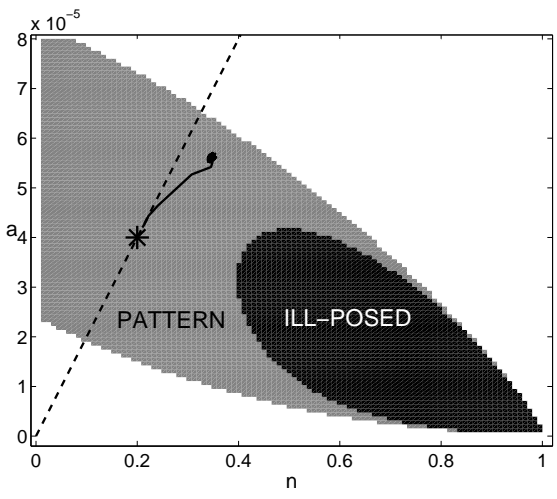

(b)

Fig. 1. Here we show how the patterning and ill-posed regions for $(24,25)$ depend on the model parameters when the chemical potential has exponential decay (35). For fixed $s_{1}$, bifurcations to spatial patterns occur along the dashed line $a=n / s_{1}$. (a) with no background potential $\left(\alpha_{1}=0\right)$, solutions that are initially within the patterned region may evolve and enter the ill-posed region; (b) when a background potential is included $\left(\alpha_{1}=0.05\right)$, there are upper and lower thresholds for patterning, and solutions may remain well-posed for all time. The asterisk indicates the spatially-uniform steady state $\left(n_{0}, a_{0}\right)=(0.2,0.00004)$ about which the system was perturbed to produce the simulations presented in Figure 2. The solid trajectory in (b) tracks the point in space closest to the ill-posed region, defined by (39), from the simulation of Figure 2(a). This illustrates that the solution does indeed remain well-posed. Other parameter values: $\beta_{1}=1, \theta_{1}=50000, s_{1}=5000$. 

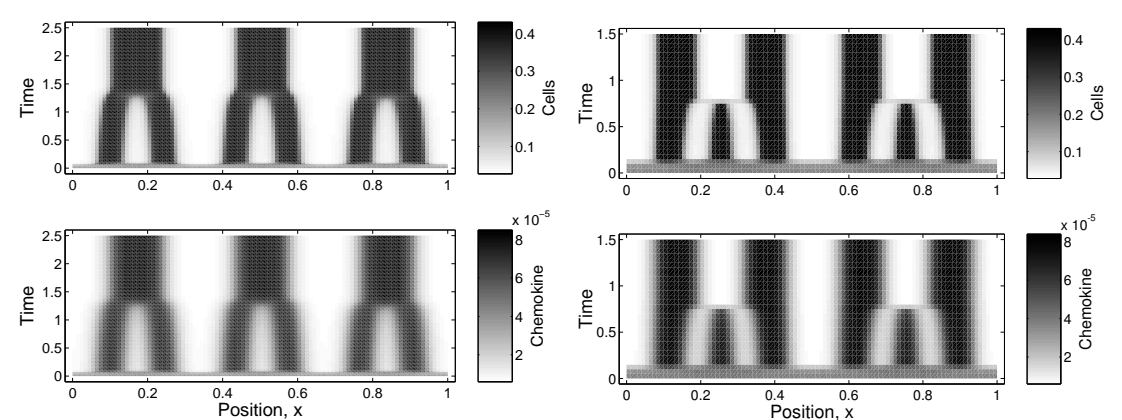

(a)

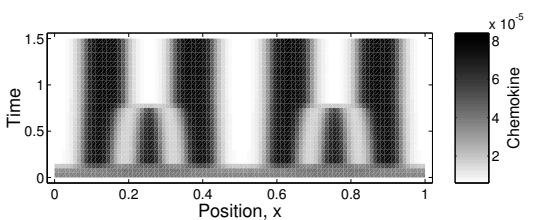

(b)

Fig. 2. Typical simulations illustrating the types of spatial patterns that emerge for different choices of the initial conditions when the chemical potential has exponential decay (35). The spatially uniform steady state $\left(n_{0}, n_{0} / s_{1}\right)$ (marked with an asterisk on Figure 1(b)) was perturbed using initial conditions of the form $n_{\text {in }}(x)=n_{0}+0.01 \cos (2 \pi x / \omega), a_{i n}(x)=n_{\text {in }}(x) / s_{1}$. (a) $\omega=0.333$ : a 3-peaked pattern emerges; (b) $\omega=0.5$ : a 4-peaked pattern emerges. These two patterns persist after 100 time units, but we cannot exclude the possibility that they eventually evolve to a common pattern. The other parameter values are as in Figure 1(b), with $n_{0}=0.2, a_{0}=0.00004$. 


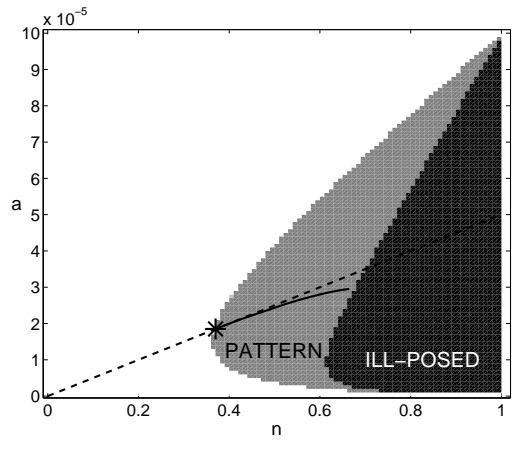

(a)

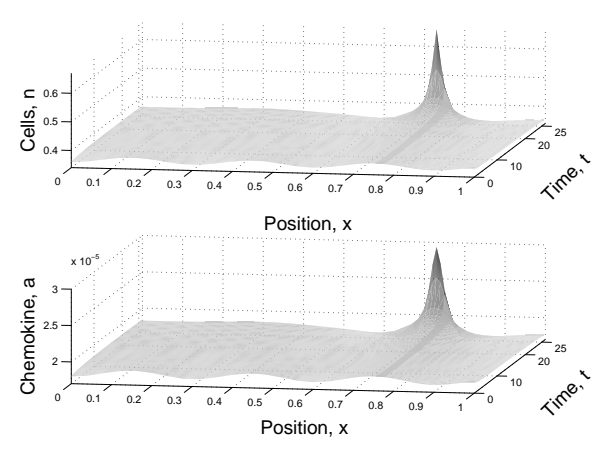

(b)

Fig. 3. Rational potential function (36) with $m=1$. (a) For $m=1$ the ill-posed region always sits inside the patterning region, intersecting at $\left(1, \beta_{2} /\left(\alpha_{2} \theta_{2}\right)\right)$ and $(1,0)$. (b) Solutions evolve from a perturbation of $\left(n_{0}=0.37, a_{0}=n_{0} / s_{1}\right)$ to give cellular aggregations, and solutions enter the ill-posed region. In (a) we include the trajectory of the point in space closest to the ill-posed region, defined by (39). It is clear that the trajectory approaches that region, leading to numerical instability at time $t \approx 25.56$. Parameter values: $\alpha_{2}=0.05, \beta_{2}=1, \theta_{2}=200000, s_{1}=20000$. Initial conditions: $n_{\text {in }}(x)=n_{0}+0.01 \cos (2 \pi x / \omega), a_{\text {in }}(x)=n_{\text {in }}(x) / s_{1}$, where $\omega=$ 0.3 . 


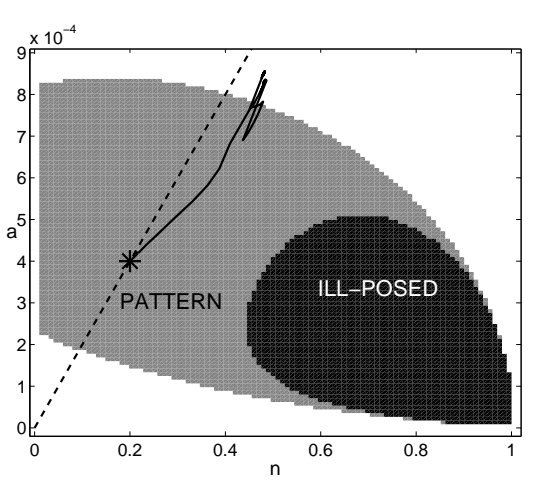

(a)
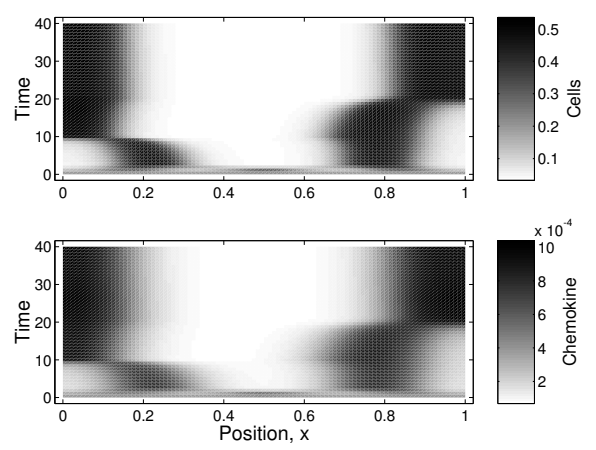

(b)

Fig. 4. Rational potential function (36) with $m=2$. (a) The line $a=n / s_{1}$ (dashed) can lie outside the ill-posed region, and patterns may develop and remain well-posed. (b) Numerical simulation showing the evolution of patterns from initial conditions $n_{\text {in }}(x)=n_{0}+0.01 \cos (2 \pi x / \omega), a_{\text {in }}(x)=n_{\text {in }}(x) / s_{1}$, where $\omega=$ 0.333. In (a) we include the trajectory of the point in space closest to the ill-posed region, defined by (39), illustrating that the solution remains within the well-posed region. Parameter values: $\alpha_{2}=0.05, \beta_{2}=1, \theta_{2}=5000, s_{1}=500, n_{0}=0.2$. 


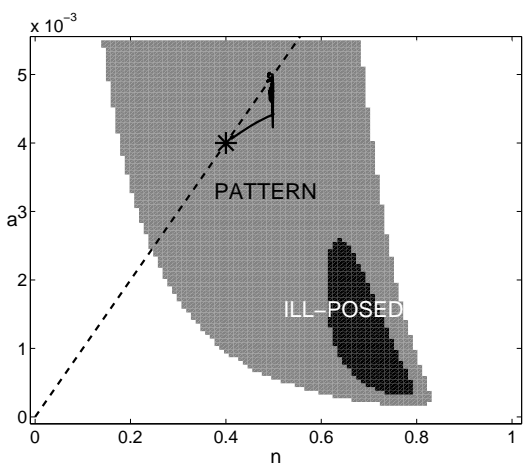

(a)

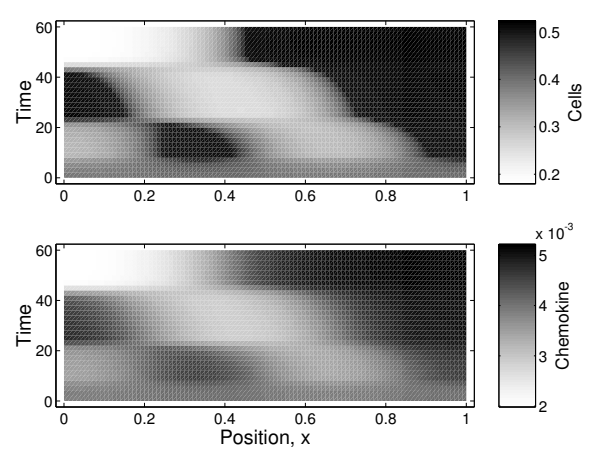

(b)

Fig. 5. Rational potential (36) plus the cellular potential (44) to maintain well-posedness $-m=1$ so that without the additional cellular potential the problem becomes ill-posed as cells aggregate. (a) Patterning and ill-posed regions. As the strength of the cellular potential, $\lambda$, increases, the ill-posed regions shrinks. (b) Numerical simulation. In (a) we include the trajectory of the point in space closest to the ill-posed region, defined by (39). Parameters: $\alpha_{2}=0, \beta_{2}=1, \theta_{2}=1000, n^{*}=0.5, \lambda=0.007, n_{0}=0.4, s_{1}=100$. Initial conditions: $n_{\text {in }}(x)=n_{0}+0.01 \cos (2 \pi x / \omega), a_{\text {in }}(x)=n_{\text {in }}(x) / s_{1}$, where $\omega=0.666$. 